\title{
ON PROGNOSIS OF INDUSTRIAL ENTERPRISE INVENTORY MANAGEMENT FOR RECOMMENDATION TO OPTIMIZE THIS PROCESS
}

\section{E. L. PANKRATOV}

Nizhny Novgorod State University

23 Gagarin Avenue

Nizhny Novgorod, 603950

Russia

Nizhny Novgorod State Technical University

24 Minin Street

Nizhny Novgorod, 603950

Russia

e-mail: elp2004@mail.ru

\begin{abstract}
The need to maximize profits leads to the need to compile an optimal output plan with available resources and to decrease costs of products [1-5]. One of the forms of costs is the cost of storing stocks. With their increase, this value also
\end{abstract}

2020 Mathematics Subject Classification: 34A25, 91-10.

Keywords and phrases: minimization of attendant costs, inventory management, modelling of economical processes, prognosis of economical processes, optimization of economical processes.

Received November 3, 2019

(C) 2020 Scientific Advances Publishers

This work is licensed under the Creative Commons Attribution International License (CC BY 3.0).

http://creativecommons.org/licenses/by/3.0/deed.en_US

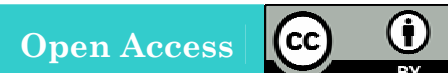


increases. At the same time, a decrease in the supply of resources can lead to a halt in production. In this situation, the present paper proposes a model of enterprise inventory management. On the basis of this model, a compromise value of the parameters was obtained, which makes it possible to minimize the costs associated with the availability of reserves.

\section{Introduction}

The need to maximize profits leads to the need to compile an optimal output plan with available resources and to decrease costs [1-5]. One of the forms of costs is the cost of storing stocks. With their increase, this value also increases. At the same time, a decrease in the supply of resources can lead to a halt in production. In this situation, the present paper proposes a model of enterprise inventory management. On the basis of this model, a compromise value of the parameters was obtained, which makes it possible to minimize the costs associated with the availability of reserves.

\section{Method of Solution}

We consider the equation of costs in the following form:

$$
I=\frac{a b}{x}+b c+d x
$$

Here $x$ is the size of the product, $a$ is the organizational cost, $b$ is the intensity of demand, $c$ is the cost of the products, and $d$ is the cost of maintaining of products. The first term of the function (1) describes the general organizational costs, the second term of the function (1) describes the cost of the products, and the third term of the function (1) describes the total costs of maintaining the stocks. Parameters $a, b, c$, and $d$ are assumed to be constant and known parameters. We determine the quantity of the consignment corresponding to the minimum in the framework of the standard procedure for finding the extremum of the function [6], i.e., from the condition that the derivative of cost function $I$ by the product quantity $x$ is equal to zero. This condition can be represented in the following form:

$$
\frac{d I}{d x}=-\frac{a b}{x^{2}}+d=0 .
$$


Solution of this equation could be written in the following form: $x_{0}=\sqrt{a b / d}$. The quantity of product corresponds to the minimum of cost of the enterprise. Dependences of the cost function (1) on the product quantity are shown in Figures 1. Figure 1(a) shows dependence of the cost function on the product quantity at different values of organizational cost $a$. Increasing of number of curves numbers corresponds to increasing of value of organizational cost. The figure shows that increasing of organizational cost leads to increasing of the considered cost function. One can find maximal difference of cost function at different value of organizational cost near optimal value of product quantity $x_{0}$. Increasing and decreasing of organizational cost leads to decreasing of difference between values of cost function with different values of organizational cost. Figure 1(b) illustrates dependence of the cost function on the product quantity at different values of intensity of demand $b$. Increasing of number of curves numbers again corresponds to increasing of value of intensity of demand. In this situation, one can find again maximal difference of cost function at different value of intensity of demand near optimal value of product quantity $x_{0}$. Increasing and decreasing of organizational cost leads to decreasing of difference between values of cost function with different values of intensity of demand. However, the difference between different values of cost function at different values of intensity of demand is larger than at different values of organizational cost. Figure 1(c) illustrates dependence of the cost function on the product quantity at different values of cost of the products $c$. In this situation one can find the similar trends as for previous figures. However in area of small values of product quantity one can find fast rendezvous of all curves. At the same time in area of large values of product quantity one can find almost parallel lines for all dependences. Figure 1(d) illustrates dependence of the cost function on the product quantity at different values of maintaining of products $d$. In this situation one can find the similar trends as for Figures 1(a) and 1(b) again. In area of small values of product quantity one can find fast rendezvous of all curves anew. However, Figure 1(d) shows essential expansion of increasing of cost function with increasing of maintaining of products. 


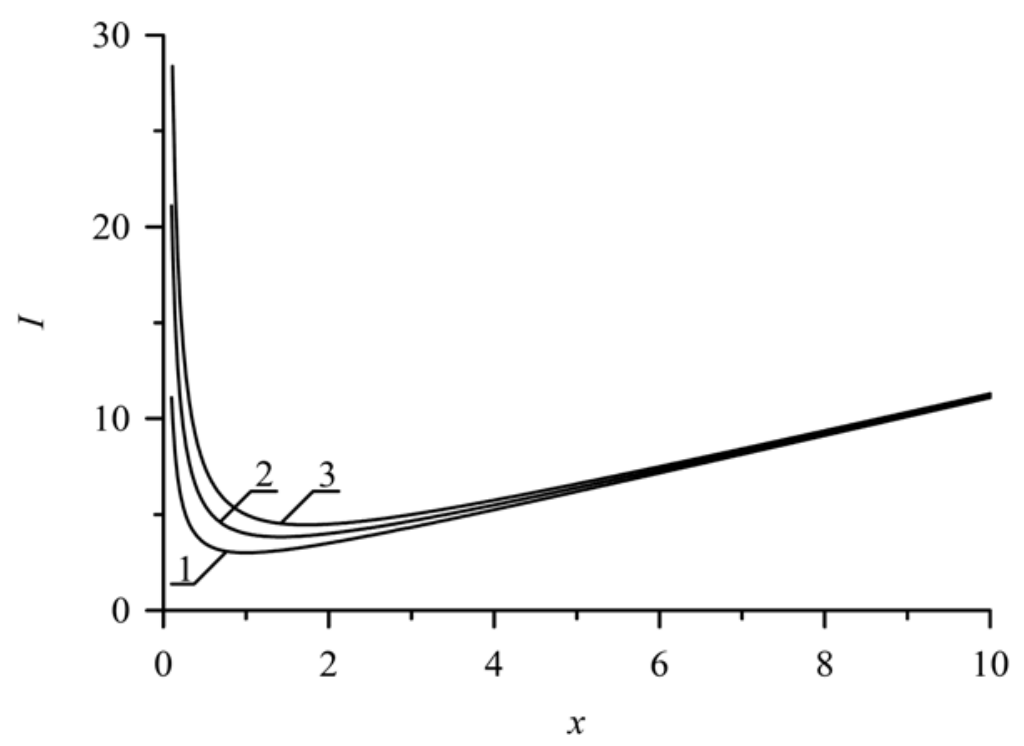

Figure 1(a). Dependence of the cost function on the quantity of product at different values of organizational cost $a$.

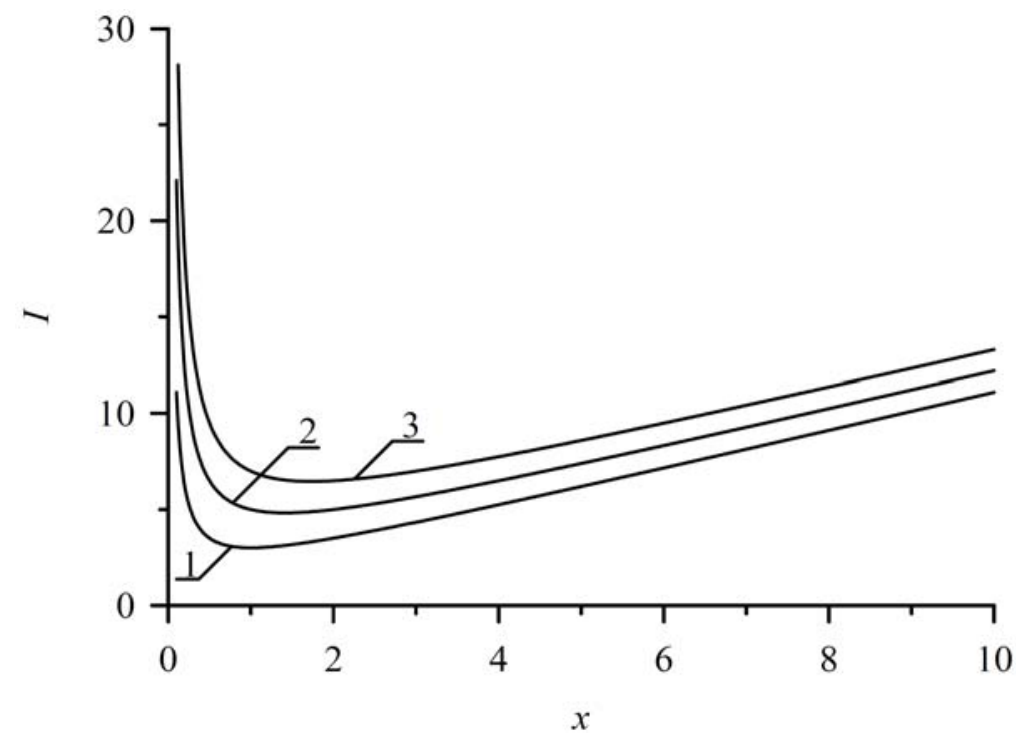

Figure 1(b). Dependence of the cost function on the quantity of product at different values of intensity of demand $b$. 
ON PROGNOSIS OF INDUSTRIAL ENTERPRISE ...

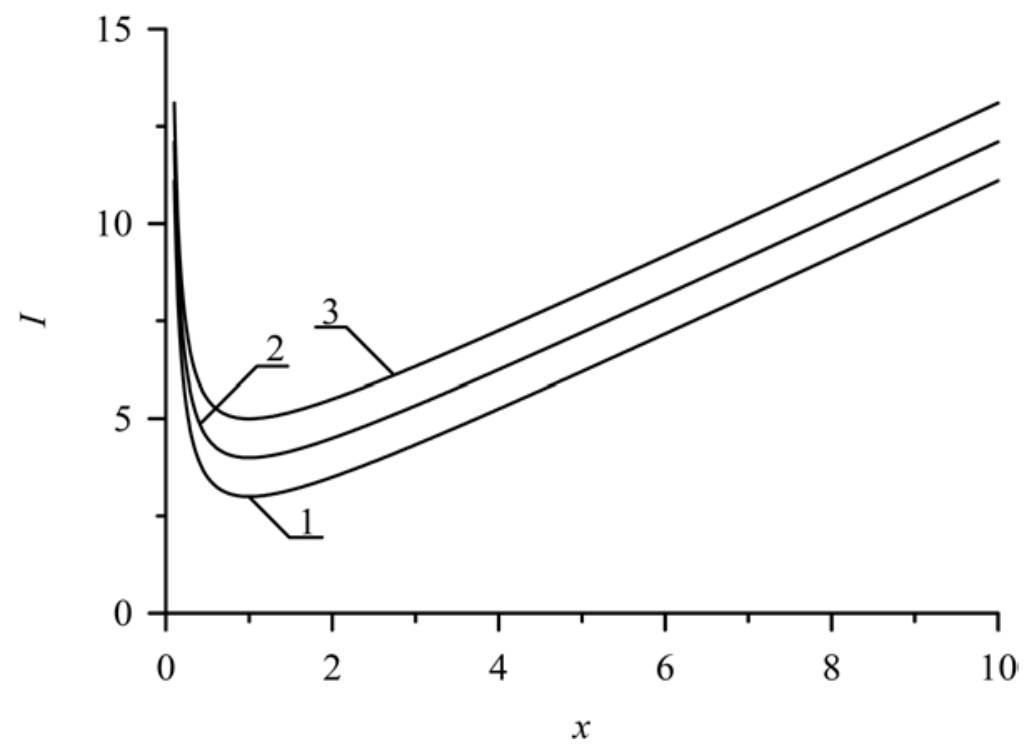

Figure 1(c). Dependence of the cost function on the quantity of product at different values of cost of the products $c$.

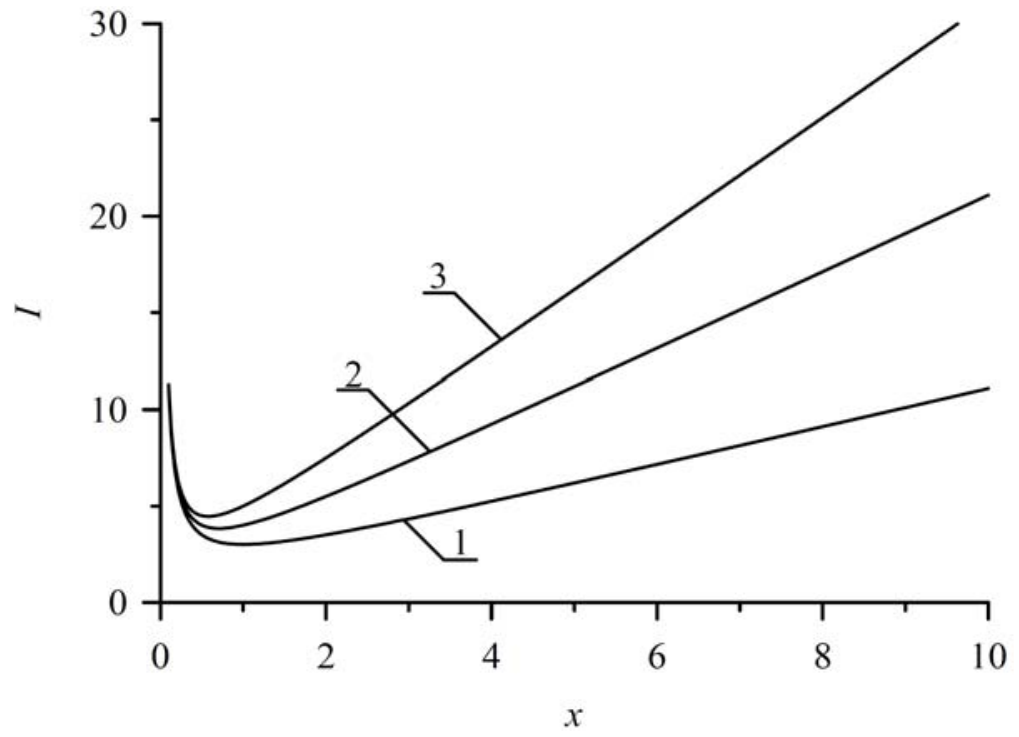

Figure 1(d). Dependence of the cost function on the quantity of product at different values of maintaining of products $d$. 
As quantitative characteristics of changing of the cost function (1) it could be used derivative of the function on the product quantity (2). Figures 2 illustrate the derivative. Figure 2(a) illustrates dependence of the considered derivative of the cost function on the product quantity at different values of organizational cost $a$. Increasing of number of curves numbers corresponds to increasing of value of organizational cost. The figure shows increasing of the cost function with increasing of organizational cost. The dependence is enough clear framework economic theory. Increasing of quantity of product $x$ before minimum of cost leads to decreasing of cost function due to increasing of profit. Increasing of quantity of product $x$ after minimum of cost leads to excess of the product in the considered industrial enterprise. Figure 2(b) illustrates dependence of the derivative of the cost function on the product quantity at different values of intensity of demand $b$. Increasing of number of curves numbers again corresponds to increasing of value of intensity of demand. Figure 2(c) illustrates dependence of the derivative of the cost function on the product quantity at different values of maintaining of products $d$. In this situation one can find the similar trends as for Figures 2(a) and 2(b). The considered derivative of the cost function on the quantity of product did not depends on the cost of the products $c$. 
ON PROGNOSIS OF INDUSTRIAL ENTERPRISE ...

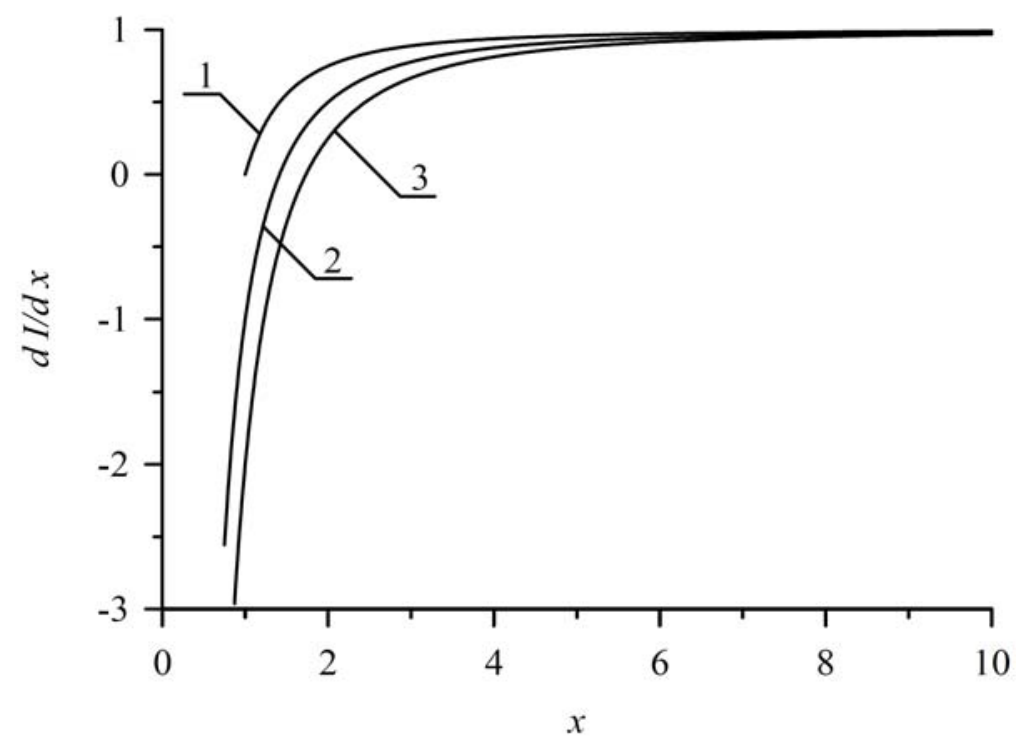

Figure 2(a). Dependence of derivative of the cost function on the quantity of product at different values of organizational cost $a$.

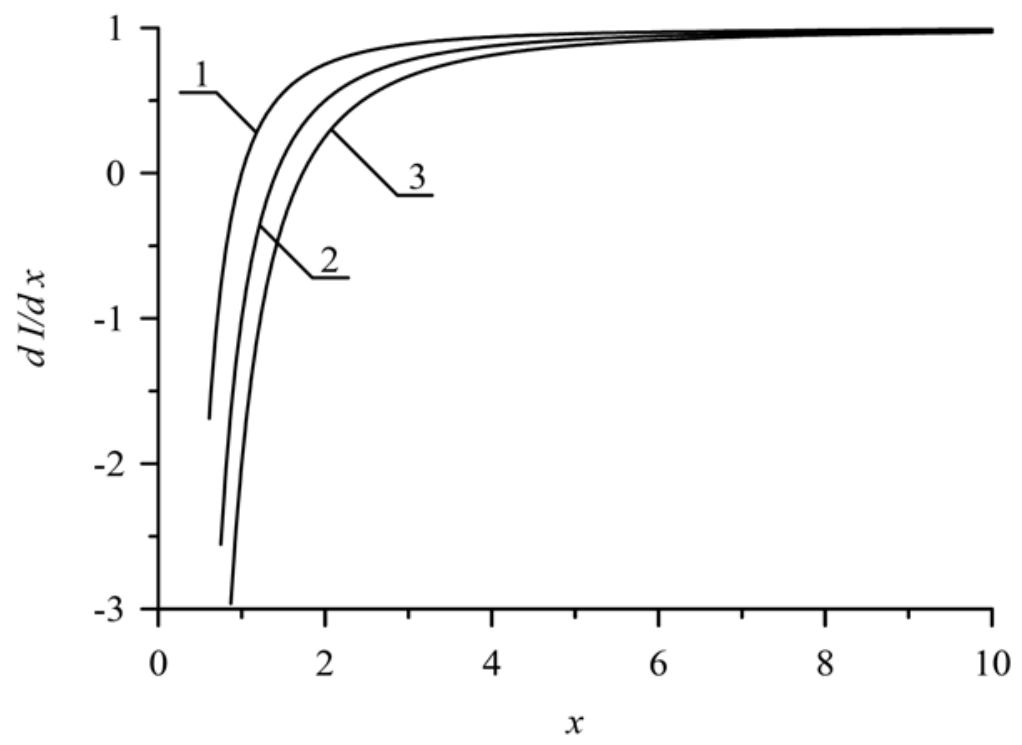

Figure 2(b). Dependence of derivative of the cost function on the quantity of product at different values of intensity of demand $b$. 


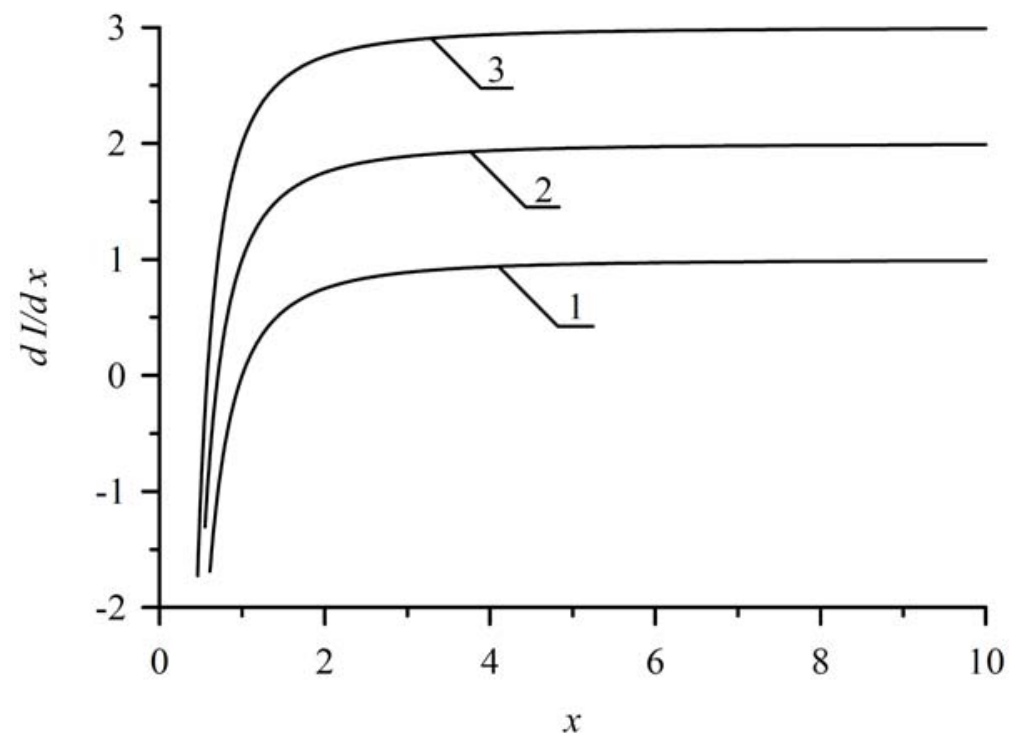

Figure 2(c). Dependence of the derivative of cost function on the quantity of product at different values of maintaining of products $d$.

Now let us analyze influence of single terms of the cost function (1) on value of the function. In this situation we neglect by two terms of the function in comparison with one of them. After that we compare of the term with original cost function (1). We illustrate the comparison by Figures 3. Figure 3(a) illustrates comparison of original cost function (1) with the first term of the function. Figure 3(b) illustrates comparison of original cost function (1) with the second term of the function. Figure 3(c) illustrates comparison of original cost function (1) with the third term of the function. 


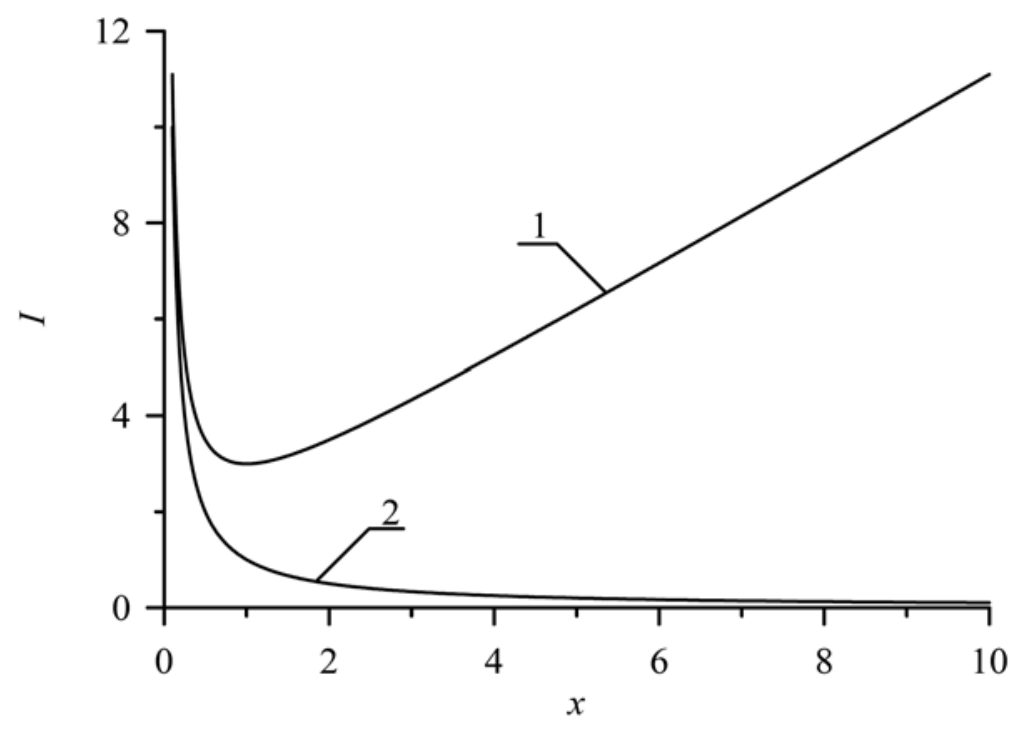

Figure 3(a). Dependence of the cost function on the quantity of product in comparison with the first term of the function.

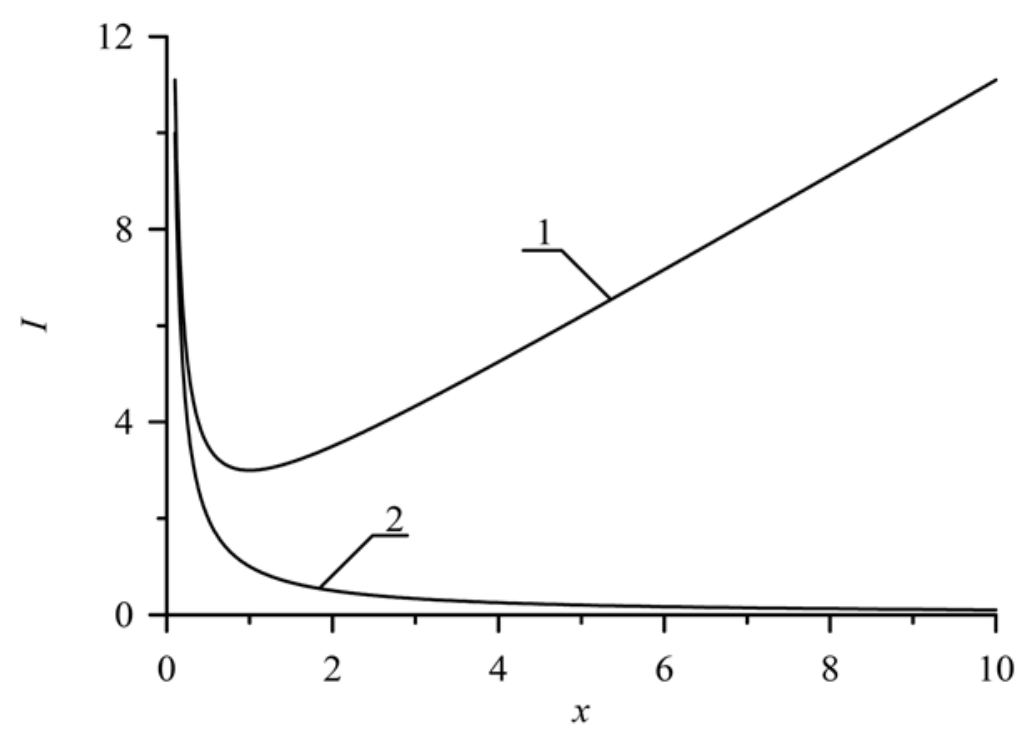

Figure 3(b). Dependence of the cost function on the quantity of product in comparison with the first term of the function. 


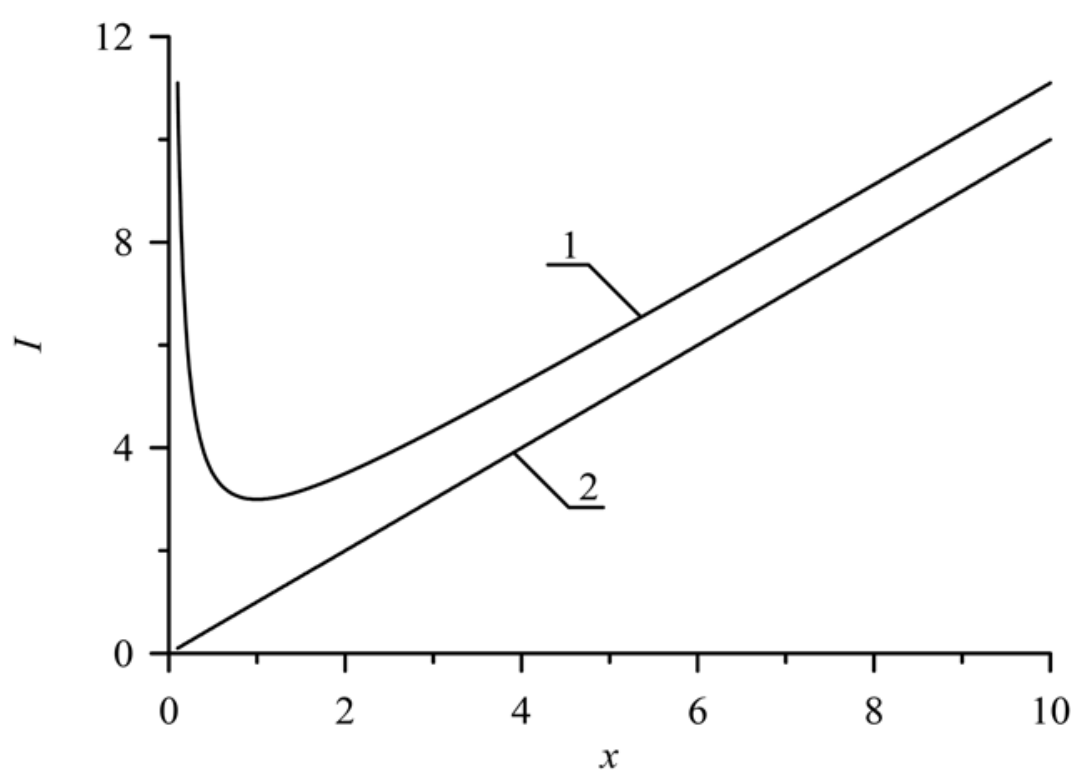

Figure 3(c). Dependence of the cost function on the quantity of product in comparison with the third term of the function.

Farther we analyze derivative of cost function on the quantity of product for the same limiting cases. Figures 4(a) and 4(b) show the derivative (2) in comparison with the first and the second terms of the function. 
ON PROGNOSIS OF INDUSTRIAL ENTERPRISE ...

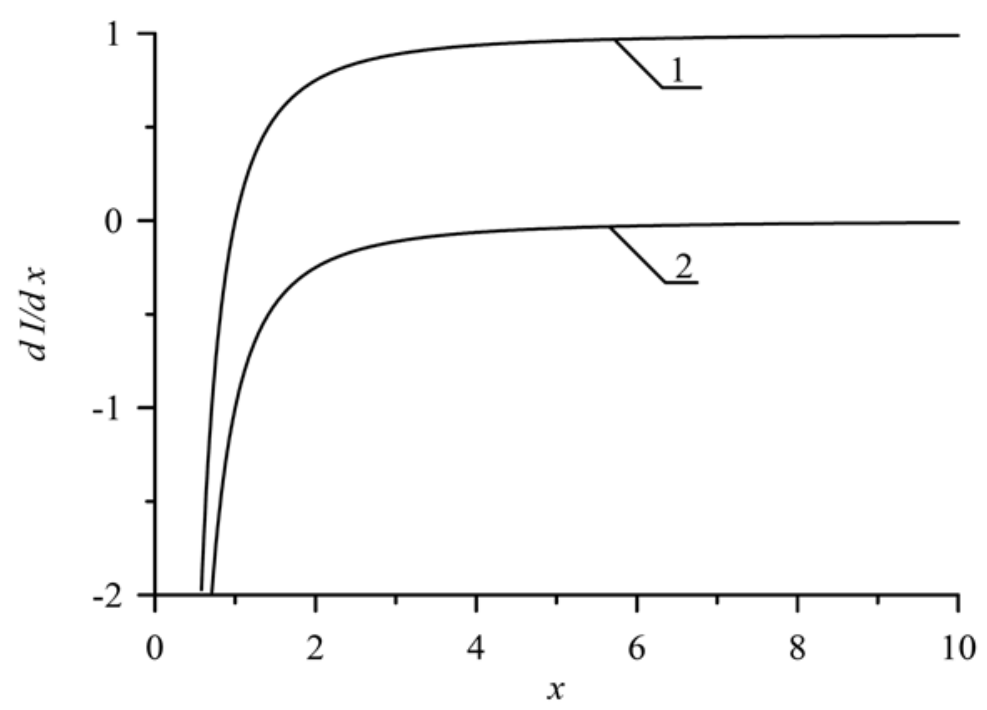

Figure 4(a). Dependence of derivative of the cost function on the quantity of product in comparison with the first term of the function.

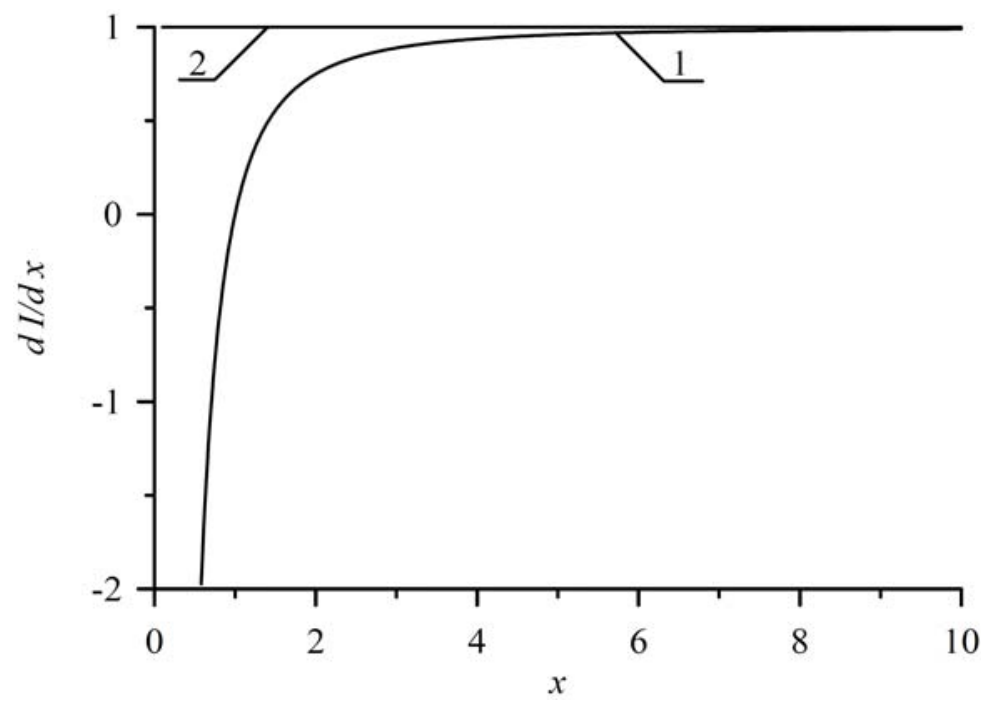

Figure 4(b). Dependence of derivative of the cost function on the quantity of product in comparison with the first term of the function. 


\section{Conclusion}

In this paper, we introduce a model for description of inventory management. Based on the model, a condition is formulated to minimize the attendant costs.

\section{References}

[1] R. Yaghoubi, M. Yaghoubi, S. Locke and J. Gibb, Mergers and acquisitions: A review (part 2), Studies in Economics and Finance 33(3) (2016), 437-464.

DOI: https://doi.org/10.1108/SEF-07-2015-0165

[2] J. Prat, Dynamic contracts and learning by doing, Mathematics and Financial Economics 9(3) (2015), 169-193.

DOI: https://doi.org/10.1007/s11579-014-0120-6

[3] C. W. Wu and W. Z. Hung, Real national income average growth rate: A novel economic growth and social fair evaluation index, Economics Research International (2010); Article ID 678927.

DOI: https://doi.org/10.1155/2015/678927

[4] St. Villeneuve and X. Warin, Optimal liquidity management and hedging in the presence of a non-predictable investment opportunity, Mathematics and Financial Economics 8(2) (2014), 193-227.

DOI: https://doi.org/10.1007/s11579-013-0110-0

[5] F. Ferriani, Traders and time: Who moves the market?, Studies in Economics and Finance 32(1) (2015), 74-97.

DOI: https://doi.org/10.1108/SEF-03-2014-0065

[6] G. Korn and T. Korn, Mathematical Handbook for Scientists and Engineers, Definitions, Theorems and Formulas for Reference and Review, Second Edition, McGraw-Hill Book Company, New York, 1968. 